\title{
RÉALISATION DE DÉTECTEURS EN GERMANIUM GOMPENSÉS AU LITHIUM
}

\author{
A. GALLMANN et M. A. MOLINARI,
}

Institut de Recherches Nucléaires, Strasbourg-Cronenbourg.

\begin{abstract}
Résumé. - Nous avons étudié par gammagraphie un certain nombre de détecteurs Ge(Li), en particulier un détecteur pseudo-coaxial, dont les performances se sont nettement améliorées après ablation de la zone mauvaise. Pour améliorer le rapport photopic/Compton des détecteurs coaxiaux, nous avons enlevé le cœur de type $\mathrm{P}$ par étincelage. Deux diodes de ce type sont présentées.

Abstract. - We have studied several $\mathrm{Ge}(\mathrm{Li})$ detectors by using the gamma-ray scanning technique. A particular example is that of a single-open end detector, the performances of which have been substantially increased after cutting the bad region. In order to improve the photopeaks-to-Compton ratio of coaxial detectors, we have used a spark machine for removing the central P-type core. Two such cases are presented.
\end{abstract}

La limitation de la résolution en énergie des détecteurs $\mathrm{Ge}(\mathrm{Li})$ tient essentiellement aux défauts des monocristaux de germanium, en particulier aux centres de capture des porteurs de charge, à leur répartition et à leur densité au sein du matériau. Le seul moyen d'in- vestigation à l'heure actuelle est de faire la gammagraphie des détecteurs, technique qui a été décrite par H. L. Malm [1], et largement utilisée par l'équipe de Chalk River [2-9]. Gette méthode est utilisée dans notre laboratoire depuis plus d'un an avec un triple 
but : i) détermination du volume utile des diodes et de la géométrie exacte des régions sensibles; ii) localisation des régions de mauvaise résolution et de faible efficacité; iii) sélection des monocristaux.

La détermination des zones perturbées d'un détecteur peut être d'un très grand intérêt dans le cas où il devient possible de supprimer ces mauvaises régions. Ges mesures nous ont permis, à plusieurs reprises déjà, d'améliorer considérablement les performances de détecteurs. Ceci a été notamment le cas d'un détecteur de $23 \mathrm{~cm}^{3}$ dont la résolution globale était de $10 \mathrm{keV}$ pour les raies de ${ }^{51} \mathrm{Cr}$. Il n'y avait aucune raison apparente pour que ce détecteur ne fonctionne pas correctement. En effet, le cristal sélectionné présentait des caractéristiques que l'on juge normalement bonnes. De plus, toutes les mesures faites sur cette diode étaient normales, à savoir faible capacité de $24 \mathrm{pF}$, champ supérieur à $100 \mathrm{~V} / \mathrm{mm}$, courant inverse à $1000 \mathrm{~V}$ de l'ordre de $10^{-3} \mathrm{~mA}$, etc. Sans nul doute, le cristal devait présenter des défauts qui ne pouvaient être mis en évidence que par une gammagraphie systématique.

Cet examen point par point à l'aide d'un faisceau $\gamma$ collimaté de ${ }^{51} \mathrm{Cr}(320 \mathrm{keV})$ nous a permis de déceler une zone perturbée, située à une extrémité du cristal et ayant une épaisseur d'environ 5 à $6 \mathrm{~mm}$. A cet endroit, le cristal présentait vraisemblablement des centres de recombinaison en raison de la perte importante d'efficacité. Après avoir enlevé la partie en question de la diode, nous avons pu constater que la résolution s'était nettement améliorée $(\sim 2,5 \mathrm{keV})$ et que la courbe d'efficacité avait l'allure normale de celle que doit avoir un bon détecteur.
Nous pensons que l'avenir de la gammagraphie n'est pas tellement dans la récupération des monocristaux. Nous la voyons plutôt comme un moyen d'investigation puissant et complémentaire dans le choix des monocristaux pour la fabrication de détecteurs de grand volume sensible.

Le fond Compton observé dans tous les détecteurs en $\mathrm{Ge}(\mathrm{Li})$ est bien souvent gênant pour la détermination de pics de faible intensité. H. L. Malm [10] a montré qu'il était possible de diminuer la diffusion Compton, donc d'accroître le rapport pic photoélectrique/Compton de $50 \%$ environ en enlevant le cœur de type $\mathrm{P}$ des détecteurs coaxiaux.

Plusieurs détecteurs de ce type ont été réalisés dans notre laboratoire. L'enlèvement du cœur a été fait par étincelage. Dans certains cas, nous avons fait cette opération après compensation de la diode. Le contact $\mathrm{P}$ a été rétabli par diffusion de Ga-In. Dans d'autres cas, l'enlèvement du cœur a été fait avant diffusion de $\mathrm{Li}^{+}$ dans le germanium.

Un détecteur de $30 \mathrm{~cm}^{3}$, coaxial percé, fonctionne actuellement depuis un an, polarisé à $1500 \mathrm{~V}$, avec une résolution de $3,3 \mathrm{keV}$ pour les lignes de ${ }^{60} \mathrm{Co}$ et un rapport pic/Compton de 11 . Un autre détecteur de $46 \mathrm{~cm}^{3}$ vient récemment d'être mis en service. G'est également une diode coaxiale percée de grande section, dont la résolution à $1800 \mathrm{~V}$ de polarisation est inférieure à $4 \mathrm{keV}$, et le rapport pic/Compton de 11 . Les résolutions indiquées sont mesurées avec une électronique classique disponible commercialement et dont l'étage d'entrée du préamplificateur n'est pas refroidi.

\section{BIBLIOGRAPHIE}

[1] MaLM (H. L.), I.E.E.E., Trans. Nucl. Sci., 1966, NS 13, 285.

[2] Chartrand (M. G.) et Malm (H. L.), AECL-2764.

[3] Sakai (E.), Marm (H. L.) et FowleER (I. L.), Semiconductor Nuclear Particle Detectors and Circuits Conf., Gatlinburg, Tenn. U.S.A., 1967, et AECL-2762.

[4] Sakar (E.), 14th Nuclear Science Symposium, Los Angeles, 1967.

[5] Sakat (E.), Appl. Phys. Letters, 1968, 12, 269.
[6] SAKaI (E.), I.E.E.E., Trans. Nucl. Sci., 1968, NS 15, 310.

[7] SakaI (E.) et FOWLER (I. L.), I.E.E.E., Trans. Nucl. Sci., 1968, NS 15, 327.

[8] WeBb (P. P.) et al., Nucl. Instr. Methods, 1968, 63, 125.

[9] Sakai (E.), McMath (T. A.) et Franks (R. G.), 15th Nuclear Science Symposium, Montréal, 1968.

[10] MaIm (H. L.), Progress Report, Physics Division, PR-P75 (3.5.4) 